\title{
Energy Efficient Hierarchy-based Clustering Routing Protocol for Wireless Sensor Networks
}

\author{
Subhrendu Guha Neogi \\ Assistant Professor and \\ Research Scholar \\ Department of CSE \\ Sir Padampat Singhania \\ University, Bhatewar, \\ Udaipur - 313601 \\ Rajasthan, India
}

\author{
Anand A. Bhaskar \\ Assistant Professor and \\ Research Supervisor \\ Department of ECE \\ Sir Padampat Singhania \\ University, Bhatewar, \\ Udaipur - 313601 \\ Rajasthan, India
}

\author{
Prasun Chakrabarti \\ Associate Professor \\ Department of CSE \\ Sir Padampat Singhania \\ University, Bhatewar, \\ Udaipur - 313601 \\ Rajasthan, India
}

\begin{abstract}
Wireless sensor network (WSN) consisting of a large number of sensor nodes is effective for gathering data in a variety of environments. Since the sensors operate on battery of limited power, it is a challenging task to design an efficient routing scheme which can minimize the delay while offering high energy efficiency and long network lifetime. Achieving both energy efficiency and scalability at the same time is a challenging task in wireless sensor networks. In this paper a new energy efficient hierarchy-based clustering routing protocol (EEHCR) is proposed for data gathering scheme for Wireless sensor networks. This is very crucial to ensure that the system operates at minimum energy with increasing scalability and network life-time. Simulation results show that the proposed routing scheme significantly reduces energy consumption and increases the lifetime of sensor network compared to other hierarchical routing protocols.
\end{abstract}

\section{Keywords}

Wireless sensor network (WSN), energy efficiency, network life-time, scalability, cluster head, clustering algorithm.

\section{INTRODUCTION}

A WSN is a specialized wireless network made up of a large number of sensors and at least one base station. The sensor nodes are small devices that consist of four basic components 1) sensing subsystem, 2) processing subsystem, 3) wireless communication subsystem 4) energy supply subsystem [1]. The sensor nodes have limited battery power, communication range and memory etc. [2]. WSN are characterized with denser levels of sensor node deployment, higher unreliability of sensor nodes and several power, computation and memory constraints [3]. Due to severe energy constraints of large number of densely deployed sensor nodes, it requires a suite of network protocols to implement various network control and management functions such as synchronization, node localization and network security [4]. With the advances in micro-electro-mechanical system technologies, embedding system technology and wireless communication with low power consumption, it is now possible to produce micro wireless sensors for sensing, wireless communication and information processing [5]. These inexpensive and powerefficient sensor nodes works together to form a network for monitoring the target region. Through the cooperation of sensor nodes, the WSNs collect and send various kinds of message about the monitored environment (e.g. temperature, humidity, etc.) to the sink node, which processes the information and reports it to the user. Sensor networks have a wide variety of applications and systems with vastly varying requirements and characteristics. Scalability and performance consistencies are the major design attributes of sensor networks. To allow the system to cope with additional load and to be able to cover a large area of interest without degradation of services, networking clustering has been pursued in some routing approach [6]. The hierarchical cluster based routing is advantageous as the scalability and the power-efficiency in the sensor network improves. In this hierarchical based architecture, nodes with higher power levels perform the fusion of data gathered from the other sensor nodes and transmit the aggregated data to the basestation (BS) while the nodes with low power levels only perform the sensing of the environment. They transmit the sensed data to the higher node, known as the cluster-heads (CHs) which are at a lesser distance than the base station. The cluster formation and the assignment of special tasks to the cluster heads $(\mathrm{CHs})$ reduce the power dissipation within a particular cluster, which improves the scalability of the sensor network [7]. Also by aggregating the sensed data, the amount of data to be transmitted to the base-station (BS) is reduced and the lifetime of the overall sensor network is increased. As the data travels from a lower cluster level to a higher cluster level, it covers more distance and the data travels faster to the base station (BS).The main objectives of clustering hierarchy is to efficiently maintain the energy consumption of sensor nodes by involving them in ad-hoc communication within a particular cluster and by performing data aggregation and fusion in order to decrease the number of transmitted message to the base station. Cluster formation is typically based on the reserve of sensors and sensors' proximity to the cluster head. Hierarchical routing protocols are critical for the wireless sensor networks (WSN) to maximize its lifetime, but the existing protocols are prone to lead nodes in clusters to die early due to ignoring the state of neighbors in the cluster head decision. The lifetime of sensor can be increased by optimized the applications, operating systems, and communication protocols. In hierarchy-based clustering, the network is randomly divided into several clusters, where each cluster is managed by a cluster head $(\mathrm{CH})$ [8]. The sensor nodes transmit data to their cluster heads, which transmit the aggregated data to the base station. Hierarchical and cluster based routing protocol for Wireless Sensor Network supports mobility in the Sensor Nodes as well as in the Sink. The entire protocol has two phases namely Setup Phase and Data Forwarding Phase. After deployment of the Sensor Nodes entire sensor field in divided into some logical clusters and each cluster contains Sensor Nodes with different roles such 
as Gateway Node, Cluster Head Node and Ordinary Sensor Node. Majority of the computation intensive tasks are carried out in the Sink [9]. In EEHCR, each cluster is managed by a set of associates and the energy efficient clusters are retained for a longer period of time; the energy-efficient clusters are identified using heuristics-based approach. Therefore, it is significant to extend the sensor networks lifetime through proficient use of the sensor nodes energy. The new proposed protocol increases the system lifetime by reducing the energy consumption.

In this paper the mechanism of energy efficient hierarchybased clustering routing protocol (EEHCR) for routing in WSN is introduced with a detailed study of other energy efficient and related routing protocols used in WSN. This paper shows the design paradigm of EEHCR and compared with other existing protocols in WSN. This paper is organized as: section 2 is concentrated on the discussion of related methods where section 3 describes the mechanism of EEHCR, section 4 gives the algorithm for the proposed techniques, section 5 with results and discussions and section 6 concludes the paper.

\section{RELATED METHODS}

A number of routing protocols for WSN have recently being developed to establish different performance metrics like energy efficiency, scalability with the optimization of routing mechanism. Routing in WSNs is generally divided in two ways: according to the network structure as flat-based, hierarchy-based, and location-based routing, and according to the protocol operation as multipath-based, query-based, and negotiation-based, QoS-based, or coherent-based [3]. This section focuses on hierarchical routing protocols, because hierarchical routing efficiently way to lowers energy consumption within a cluster, performing data aggregation and fusion to reduce the number of messages sent to the BS. The key responsibility of wireless sensor network is to forward the sensing data gathered by sensor nodes in the sensing fields to the BS. One simple approach to the fulfillment of this task is direct data transmission. In this case, each node in the network directly sends sensing data to the BS. As observed in LEACH [10], the direct approach would work best if the BS is located close to the sensor nodes or the cost of receiving is very high as compared to the cost of transmitting data. However, if the BS is remote from the sensor node, the node will soon die for suffering excessive energy consumption for delivering data. To solve this problem, some algorithms that are aimed to save energy have been proposed one after another. LEACH protocol is hierarchical routing algorithm that can organize nodes into clusters collections. Each cluster controlled by cluster head. Cluster head has several duties. First one is gathering data from member cluster and accumulates them. Second one is directly sending accumulation data to sink.

Heinzelman, et al. [11] introduced a hierarchical clustering algorithm for sensor networks, known as Low-Energy Adaptive Clustering Hierarchy (LEACH). LEACH is a cluster-based protocol that applies randomized rotation of the cluster heads to distribute the energy load evenly among the sensor nodes in the network. The operation of LEACH is organized in rounds, each consisting of a set-up phase and a steady-state phase. During the set-up phase, the network is separated into clusters, each with a randomly selected cluster head from nodes in a cluster. During the steady-state phase, the cluster heads gather data from nodes within their clusters respectively, and fuse the data before forwarding them directly to the sink. LEACH provides sensor networks with many good features, such as clustering-based, localized coordination and randomized rotation of cluster-heads, but expends much energy in cluster heads when directly forwarding data packets to the sink.

Lindsey et al. [12] presented an enhanced LEACH protocol. The protocol, Power Efficient Gathering in Sensor Information Systems (PEGASIS), assumes that all nodes have location information about all other nodes, and that each can send data directly to the base station. Hence, the chain of PEGASIS is constructed easily using a greedy algorithm based on LEACH. Each node transmits to and receives from only one of its neighbors. In each round, nodes take turns to be the leader on the chain path to send the aggregated data to the sink. To locate the closest neighbor node in PEGASIS, each node adopts the signal strength to measure the distance of all neighbor nodes. However, the global information of the network known by each sensor node does not scale well and is not easy to obtain. Since a sensor network generates too much data for the end-user to process, it has to aggregate the data.

Energy consumption is one of the most important criterions for the development of autonomous sensor network nodes. To improve efficiency all the sensor network mote designs used duty cycling techniques which means unused motes go to sleep mode with periodic wake up to save power. Battery replacement is not an option for networks with thousands of physically embedded nodes used in technologies to save power such as power-aware computing, energy-aware software or power management radios [13-14]. TEEN protocol adopts the same clustering model used by LEACH, a cluster node in TEEN sends threshold value to its members for reducing delay of transmission [15]. Power Efficient Data Gathering and Aggregation in Wireless Sensor Networks (PEDAP) [16] is based on a minimum spanning tree. PEDAP assumes that the sink knows the locations of all nodes, and that the routing information is calculated by Prim's algorithm with the sink as the root. PEDAP prolongs the lifetime of the last node in the system while providing a good lifetime for the first node. Additionally, sensor nodes transmit the sensed data to the sink via the previously constructed routing path to produce a minimum energy consuming system. Nevertheless, the intermediate nodes consume energy quickly. In the Hierarchy-Based Anycast Routing (HAR) Protocol for Wireless Sensor Networks [17], the sink constructs a hierarchical tree by sending packets (such as CREQ, CREP, CACP, PREQ) to discover each node's own child nodes in turn. HAR avoids both flooding and periodic updating of routing information, but needs to reconstruct the tree when nodes fail or new nodes are added. The drawback of HAR is that it sends and receives too many packets in the network, expending much energy. Yu and Song [18] proposed an Energy-Efficient Chain-Based routing protocol (EECB) that is an improvement over PEGASIS. EECB uses distances between nodes and the base station and remaining energy levels of nodes to decide which node will be the leader that takes charge of transmitting data to the base station. Also, EECB adopts distance threshold to avoid formation of Long Link on the chain [19]. Moreover, sensor nodes in PEGASIS from a chain and each sensor node communicate with the closest neighbors. Eventually, the chain head transmits the data to the sink and outperforms in comparison to other protocols under different network sizes and topologies [12]. Hong et al. [20] proposed an improved longest prefix matching routing algorithm. The network prefixes and the destination addresses are transformed into the decimal system and the network prefixes are stored using Scalable Bloom Filter and the destination addresses are stored segmentally to 
reduce the number of filters. Fast lookup speed is achieved by equitable distribution of the address prefixes. Several researchers devised different energy-efficient model and also tried to improve existing models of calculating energy efficiency in hierarchical clustering architecture [21-24]. The basic model is facing challenges in both setup and steady phase for electing cluster head and deciding the factors involved in energy dissipation to find shortest path for routing. Accordingly, a new energy efficient hierarchy-based clustering routing protocol (EEHCR) is proposed to overcome these issues. In EEHCR, the sensor network is constructed as a layered network like others but using different clustering approaches. The proposed design can disperse the energy consumption in the network more efficiently and prolong the network lifetime.

\section{ENERGY EFFICIENT HIERARCHY- BASED CLUSTERING ROUTING}

Energy Efficient Hierarchy-based Clustering Routing Protocol (EEHCR) is to generate energy-efficient clusters for randomly deployed sensor nodes, where each cluster is managed by a set of associates called a head-set. First step in this approach is to setup the cluster and form hierarchical clustering model. In this most efficient node becomes cluster head $(\mathrm{CH})$ in each cluster using election algorithm. Since the role of $\mathrm{CH}$ is energy consuming the election algorithm is also based on the energy parameter. After a specific number of transmissions, a set of new clusters are formed. The clusters are maintained for a short duration called round. A round consists of an election phase and data transmission phase. In an election phase, the sensor nodes self-organize into a set of clusters, where each cluster contains a head-set. In data transmission phase, the cluster heads transmits the data to base stations (BS) periodically and spontaneously. In EEHCR clustering is one of the key processes to perform routing. Clustering includes partitioning stage and choosing cluster head using election algorithm called election phase. In data transmission phase $\mathrm{CHs}$ construct route towards BS for routing which prolongs network life-time. Different phases in this model of system are (I) Cluster formation by electing cluster head (CH), (II) Hierarchical tree building, (III) Path building where cluster heads, sink will finalize the path for routing, (IV) Sensing and relaying where source can send data to sink by least cost path, (V) Tree update which may involve cluster reformation with new cluster head selection, (VI) Route maintenance to prolong the network life-time. So basically the entire process has two subgroups which are setup phase and steady phase. In setup phase the clustering and tree formation takes place where in steady phase data transmission and routing performed by the nodes in the network.

\subsection{Election Phase}

During an election phase, clusters are created by electing cluster head $(\mathrm{CH})$ in each cluster. The election of cluster head among fellow nodes for a cycle is based on battery power which needs to be broadcast among others. This information advertises initially to compare the amount of energy stored in each node and the energy efficient node elected as cluster head $(\mathrm{CH})$. Once the cluster heads are formed other nodes goes to sleep, as they do not take part in routing and required to conserve energy. This method use sleep and wake-up algorithm to decide the mode of the nodes and using this method each node goes to sleep and wake-up when required. After one round if required election process elect new $\mathrm{CH}$ in each cluster. Usually the battery power consumed with transmission but it can save energy once it goes to sleep mode. During election a weighted sum method is used for deciding the most efficient node in the cluster. The weights are assigned to the node to satisfy the following equation:

$\Sigma \mathrm{W}_{\mathrm{i}} * \mathrm{~J}_{\mathrm{i}}=1$ and $\mathrm{W}_{1} * \mathrm{~J}_{1}+\mathrm{W}_{2} * \mathrm{~J}_{2}=1$

Where, $\mathrm{W}_{1}=\mathrm{W}_{\mathrm{b}}$ is the weight given to the battery of the node and $\mathrm{W}_{2}=\mathrm{W}_{\mathrm{d}}$ is the weight given to the distance; $\mathrm{J}_{\mathrm{i}}$ is the quantity multiplied to the weights of $\mathrm{i}$-th factor

Scheduling policy is used to find out how to forward the packet from source to destination. Sleep and Wake-up scheduling is used to find out when the nodes are wake-up. This sleep and wake-up scheduling is used to increase the lifetime of sensor nodes. Asynchronous sleep wake up each node wake up independently of neighboring nodes in order to save energy. This approach is responsible uniquely balancing the work between the nodes according to set conditions that effectively result in the formation of a node that is much stronger among others in terms of battery and distance. In EEHCR sensors are organized into clusters and one node in each cluster acting as cluster-head takes the responsibility to collect data, aggregate data and finally transmit data to the distant Sink. At the end of election phase, each head-set member checks if it has sufficient energy for next round. After data transmission if required the new cluster is formed and election process takes place accordingly.

\subsection{Data Transmission Phase}

During data transmission phase, the member nodes transmit data to their $\mathrm{CHs}$ and the $\mathrm{CHs}$ transmit aggregated data to the base station (BS). First, member nodes transmit data to neighbors and second, $\mathrm{CH}$ receives the messages from the member nodes. As head-set members become $\mathrm{CH}$, only $\mathrm{CH}$ receives the messages and the remaining head-set members turn off their radio and goes to sleep mode. Third, $\mathrm{CH}$ transmits the aggregated data to BS. Fourth, $\mathrm{CH}$ checks the remaining energy and sends it to neighbors. Once the required energy calculated is higher than remaining energy it will call for election process and the $\mathrm{CH}$ removes itself from head-set. Fifth, the outgoing $\mathrm{CH}$ informs the new $\mathrm{CH}$ and sends all it message so that the new $\mathrm{CH}$ can continue the process and the remaining head-set members update it accordingly.

At the end of each round it is not necessary to reform the cluster but if any node wants to leave any head-set and join another cluster then it is easy to redesign the clusters whereas $\mathrm{CH}$ may be elected at the end of each round due to energy factor of the node. Sensor nodes can start disseminating the sensed data to the sink via the parent node. The packet format is as follows: (Seq_No, Source_ID, Dest_ID, Sink_ID, Data_Length, Payload). The Seq_No field is the sequence number of the packet and Source_ID, Dest_ID, Sink_ID fields respectively is the source node of the packet, the destination node of the packet, the sink node that requests the data packet. The Data_Length field denotes the packet length and the payload field are used to carry the data. A receiver acknowledgement packet is sent when the data packet is successfully transmitted to the parent node. The parent node then replies with this packet to notice the source node and forwards the data packet to the next hop. Each node performs the same until the data packets send to sink node via clusterhead. The data packet can be forwarded to the sink via many paths. The life-time of the network system can be extended if the sensor node always uses a different path to send data packets. Cluster dynamics describes the selection of number of clusters and formation of hierarchical tree in any particular network. In some cases the number of nodes in each cluster and number of clusters in the network can be pre-determined and in some cases it is dynamic depending on the position of 
nodes and distance between them. The number of clusters can also be dynamic but the overhead can be higher in that network during the cluster formation and designing the tree in the topology.

\subsection{Proposed Model}

This section describes the model by explaining the routing mechanism with all the factors required to calculate the energy at different stages. Before routing first work is to elect cluster-heads from each cluster for the sensor network depending on the election algorithm which may vary for every round of election. Each cluster in the network has unique cluster id. Then data will be transmitted from nodes to the respective cluster-head $(\mathrm{CH})$ and these cluster head takes part in data transmission and finally transmits it to sink. Rest other nodes in each cluster are in sleep mode to conserve energy. As only cluster heads takes part in routing of data from source to sink, each node in the cluster sends all its data to cluster-head in each round. The weighted sum method is also used for determining the routing conditions. Various considerations are followed in the proposed the network model and sensor nodes.

1. The sink and source are placed at opposite ends

2. The sensor nodes are randomly deployed in cluster

3. All nodes are of same types of sensor node

4. Data need to be collected together for sending it to cluster-head

The election of a $\mathrm{CH}$ among the fellow nodes for a cycle is based on battery power and the distance from the sink of each node. The transmission depends on the energy of the node and distance between $\mathrm{CH}$ and $\mathrm{BS}$ with nodes depending on the following factor on a weighted basis.

$\mathrm{T}_{\mathrm{c}}=\mathrm{W}_{1} * \mathrm{D}+\mathrm{W}_{2} * \mathrm{E}_{\mathrm{f}}$

where $T_{c}$ is transmission criterion, $W_{1}$ and $W_{2}$ are the weight factors; $\mathrm{D}$ is the proximity factor on the given distances of the node with $\mathrm{BS}$ and $\mathrm{CH}$ whose $\mathrm{T}_{\mathrm{c}}$ will be calculated.

The energy dissipated during transmission and reception using the following formula:

$E_{T_{X}}(k, d)=\left\{\begin{array}{cc}E_{\text {elec }} * k+\xi_{\text {fs }} * k * d^{2}, & d<d_{0} \\ E_{\text {elec }} * k+\xi_{m p} * d^{4}, & d \geq d_{0}\end{array}\right.$

$E_{R_{x}}(k, d)=E_{\text {elec }} * k$

where $E_{T x}$ is the amount of energy consumed by each node, $E_{R x}$ is the amount of energy for receiving $k$ bit packet, $E_{\text {elec }}$ is the energy dissipated, $\xi_{\mathrm{fs}}$ is the free space propagation, $\xi_{\mathrm{mp}}$ is the multiple fading channel parameter, $\mathrm{d}$ is the transmission distance and $\mathrm{k}$ is message length and $\mathrm{d}_{0}$ is the initial value of $\mathrm{d}$ where $\mathrm{d}_{0}$ and $\mathrm{d}$ are calculated as:

$d_{0}=\frac{\xi_{f s}}{\xi_{m p}}$

$d=\sqrt{\left(x_{2}-x_{1}\right)^{2}+\left(y_{2}-y_{1}\right)^{2}}$

where $\left(\mathrm{x}_{1}, \mathrm{y}_{1}\right)$ and $\left(\mathrm{x}_{2}, \mathrm{y}_{2}\right)$ are the coordinates of reference nodes.

A simple combination of different routing metrics [9] used to determine the path cost using following equations:

$\phi=\phi^{\prime}+\alpha_{1} * h^{\prime} p_{i}+\alpha_{2} * w_{i}+\alpha_{3} *$ delay $+\alpha_{4} * E_{i}$

$\phi=\phi^{\prime}+\Sigma \alpha_{i} *$ metric $_{i}$

Where $\phi^{\prime}$ is the accumulated cost along the path with different path cost metrics, metric $i_{i}$ is scaled value from $(0,1)$ and $\alpha_{1}$ is the weight factor for metric ${ }_{\mathrm{i}}$ to calculate the cost. Here, hop $\mathrm{p}_{\mathrm{i}}$ is hop count set to 1 initially, $\mathrm{E}_{\mathrm{i}}$ denotes the surplus energy, $\alpha_{\mathrm{i}}$ values are different sets of weight factors set as per requirements as per applications and $w_{i}$ calculated as per the energy consumption of node $i$ as per the following equation:

$w_{i}=e_{s}+e_{g}+e_{r}+e_{i j}$

where $e_{i j}$ calculated as per the power consumption of node $i$ transmitting data to node $j$ where $e_{g}$ is the value of generating data, $e_{r}$ is the value of receiving data and $e_{s}$ is the value of idle power consumption of node.

\subsection{Routing Protocol}

In routing protocol proposed in this method, the locations of two nodes are required to calculate the distance and to locate the nodes. In beginning all the receivers turn up their receivers. Both BS and source and sink nodes send messages about their location information. According to the node position clusters are formed and the tree is build. Nodes near the optimal position competed to be cluster head by the factors involved in election algorithm. $\mathrm{CH}$ sends the message to tell the other node within the range to join the cluster. The neighboring nodes send "ACK" message to join the cluster. If any node receives more than one request it is free to join any cluster depending on the energy cost and distance but once it joins the cluster it be member through the rest of the routing process. In the beginning of every round, all the nodes must turn up their receiver to get information from $\mathrm{CH}$. During routing each cluster member transmit the sensing data to the cluster head and finally cluster head transmit the data directly to the BS.

This protocol provides solution to the isolated nodes connections to avoid the great amount of energy is consumed by using the way that the cluster heads $(\mathrm{CHs})$ transmit their data directly to the base station with help of other CHs. The following method introduced to construct the links between isolated clusters. First, cluster head broadcast the message for requesting the distance of the node from base station from its neighboring nodes. The neighboring node which has smallest distance will be elected as relaying node can have any of the two states:

1. The selected relaying node is belonging to the other cluster. In such situation, the $\mathrm{CH}$ can forward the aggregated data smoothly to the base station with the picked up relaying node without altering the original transmission direction of the picked up node.

2. The picked up relaying node is belonging to the same cluster as the $\mathrm{CH}$ locates. In such situation, the picked up relaying node must alter its original transmission direction so that $\mathrm{CH}$ can transmit the data directly to the base station.

After the formation of routing structure, the data dissemination phase begins. In this phase of the routing protocol, sensor nodes send the data with their relaying nodes in hop-by-hop manner. To reduce the energy which is consumed in the formation of routing structure, the long time interval for this phase need to specified. After the expiration of this duration, the structure creation phase starts.

\section{EEHCR ALGORITHM}

The algorithm is explained in the following steps:

Step 1: Initialization 
The main goal of EEHCR is to build a hierarchical routing tree which can maximize the data aggregation and balance the energy consumption to enhance the network life-time. The following roles are considered for building routing tree.

I. Collaborator: It is node which detects an event and reports the collected data to the coordinator node.

II. Coordinator: It is collaborator node detects an event but after using election algorithm it becomes a coordinator and responsible for aggregating the collected data received from other collaborator and send results to the sink node.

III. Relay node: This node which is in route between coordinator and sink node and forwards the data to the link.

IV. Sink node: This node is interested in receiving the data from set of coordinator and collaborator node.

It also defines the state of the node as follows:

$$
\operatorname{state}(u)=\alpha \frac{E_{R}}{E_{\max }}+\beta \frac{M_{R}}{M_{\max }}+\gamma \frac{1}{L+1}
$$

where $\alpha+\beta+\gamma=1$ and $\mathrm{E}_{\mathrm{R}}$ is the residual energy of node $u, E_{\max }$ is the maximum energy of nodes, $M_{R}$ is the residual memory of node $\mathrm{u}, \mathrm{M}_{\max }$ is the maximum memory of nodes, $\mathrm{L}$ is the link packet loss rate of $\mathrm{u}$.

Set number of CREP $=0$ and status of parent node, CREP send and received, CREQ send and receive as NULL.

Choose BS as parent (node), BS broadcasts CREQ packets; Sensor broadcasts PREQ packets, call election algorithm to select cluster-head $(\mathrm{CH})$.

\section{Step 2: Formation of tree}

Select cluster-head $(\mathrm{CH})$ near the sink (BS) and if node elected as $\mathrm{CH}$ connect to $\mathrm{BS}$ and send request to nodes else node will join $\mathrm{CH}$ as leaf in the tree as source node. A tree rooted at Sink is formed after deploying sensor nodes, with the shortest paths (in hops) that connect all source nodes to the Sink while maximizing the possible data aggregation and balancing energy consumption. Each node has two parameters: HTT (Hop-To-Tree) and HTS (Hop-To-Sink), recording the minimum number of hops from the node to the tree and Sink respectively. HTT of the Sink and any node is initially set to 0 . The value of HTT for any node will change depending on the updating of tree while HTS remains same for each node. When Hop Configuration Message (HCM), specified as <Type, ID, HTT, State>, is received by any node which announces better distance then HTT value is changed accordingly. For each node u HCM message is received. The next hop of node $u$ is stored in $\mathrm{NH}(\mathrm{u})$. Finally $\mathrm{u}$ transmits the HCM message to its neighbors. The algorithm for tree building described as below:

1. The Sink floods an HCM with HTT=1

2. For each node $\mathrm{u}$ that received an HCM message

3. If $\mathrm{HTT}(\mathrm{u})>\mathrm{HTT}(\mathrm{HCM})$

4. $\mathrm{NH}(\mathrm{u})=\mathrm{ID}(\mathrm{HCM})$

5. $\mathrm{HTT}(\mathrm{u})=\mathrm{HTT}(\mathrm{HCM}) ; \operatorname{ID}(\mathrm{HCM})=\operatorname{ID}(\mathrm{u})$; $\operatorname{HTT}(\mathrm{HCM})=\operatorname{HTT}(\mathrm{u})+1$; State $(\mathrm{HCM})=$ State (u);

6. U transmits HCM message to its neighbors

7. Else if $(\mathrm{HTT}(\mathrm{u})==\mathrm{HTT}(\mathrm{HCM}) \& \operatorname{State}(\mathrm{NH}(\mathrm{u}))<$ State(HCM))

8. $\mathrm{NH}(\mathrm{u})=\mathrm{ID}(\mathrm{HCM})$.
Step 3: Topology model

$>$ All sensor nodes are started with same initial energy with transmission distance $\mathrm{d}_{0}$

$>$ Each sensor node can compute the distance $\mathrm{d}$ of the source based on the received location information

$>$ Transmitting power of a sensor node is controllable, i.e., transmitting power of a sensor node can be modulated according to the transmitting distance

$>$ Change the flag values accordingly as per transmission and buffer the packets for transmission

Step 4: Cluster formation

When an event occurs, a cluster based on the nodes which detect it will be formed and the election algorithm elects a Coordinator for each cluster which is Cluster Head $(\mathrm{CH})$ and deliver Cluster Configuration Message (CCM), identified as $<$ Type, ID, HTT, State, $\mathrm{W}_{\mathrm{i}}$ >, where ID is the identifier and $\mathrm{W}_{\mathrm{i}}$ is the energy factor for each node $u$. The $\mathrm{CH}$ has the maximum energy among all the nodes in the cluster. The algorithm for cluster formation described as below:

1. For each node $\mathrm{u}$ that detected the event

2. Role $(\mathrm{u})=$ Coordinator

3. u creates an CCM and broadcasts it;

4. For each node $\mathrm{u}$ that received a CCM of same event

5. If $\mathrm{HTT}(\mathrm{CCM})<\mathrm{HTT}(\mathrm{u}) \& \& \mathrm{~W}_{\mathrm{i}}(\mathrm{CCM})<\mathrm{W}_{\mathrm{i}}(\mathrm{u})$

6. $\operatorname{Role}(\mathrm{u})=$ Collaborator

7. U transmits the CCM;

8. else if $\operatorname{HTT}(\mathrm{CCM})==\operatorname{HTT}(\mathrm{u})$ \& State $(\mathrm{CCM})>\operatorname{State}(\mathrm{u}) \& \mathrm{~W}_{\mathrm{i}}(\mathrm{CCM})==\mathrm{W}_{\mathrm{i}}(\mathrm{u})$

9. $\operatorname{Role}(\mathrm{u})=$ Collaborator

10. U transmits the CCM;

11. else a new node is elected

Step 5: Path Building

Each $\mathrm{CH}$ starts establishing a path with the help of Path Building Message (PBM), Forced Path Building Message (FPBM) with two parameters <Type, Path> and Path is recorded from the message of the node $\mathrm{v}$ to the Sink where node is the $\mathrm{CH}$. The algorithm for path building described as below:

1. If $\mathrm{HTT}(\mathrm{v})==0$

2. There is no need to build path

3. Else if Energy $(\mathrm{NH}(\mathrm{v}))<$ Threshold_Energy \& Node $\mathrm{v}$ can find a neighbor who satisfies; (A) its residual energy is greater than Threshold_Energy, (B) with less HTS.

4. $\quad \mathrm{v}$ informs the nodes within event field to enter the forced path building phase

5. Else

6. $\mathrm{v}$ appends itself to the Path and sends a PBM message to its next hop;

7. For each node $\mathrm{u}$ that received a PBM message

8. If $\operatorname{HTT}(\mathrm{u})==0$

9. Path building Successful;

10. Else

11. Repeat 3 to 6 ;

12. The node with similar HTS and best state with highest energy chosen as new $\mathrm{CH}$

13. $\mathrm{NH}(\mathrm{u})=\mathrm{v}, \mathrm{v}$ is a neighbor of $\mathrm{u}$ with less HTS;

14. $\mathrm{u}$ appends itself to the Path and sends FPBM to its next hop;

15. For each node $w$ received an FPBM message

16. If $w==$ Sink

17. Forced Path building Successful;

18. Else 
19. w acts similarly to the behaviors and go to 13 ;

20. End.

\section{Step 6: Update energy of each sensor node}

The transmission depends on the energy of the node and distance between $\mathrm{CH}$ and $\mathrm{BS}$ with nodes depending on the following factor on a weighted basis.

Step 7: Each node transmit data during their allocated time slot $\mathrm{t}$ and finally data will be transmitted to $\mathrm{BS}$ via $\mathrm{CH}$
Each time after selection of cluster-heads $(\mathrm{CH})$ the information will broadcast in the cluster so that each node can send PREQ to establish connection with $\mathrm{CH}$ but in EEHCR the broadcast process will be once to minimize the overhead and utilize less energy. Fig.1 demonstrates the EEHCR model where Gateway nodes are relay nodes and data is transmitted within the cluster. EEHCR also reduces the overhead by minimizing the hop in routing and avoid periodic update to reduce the traffic in the network.

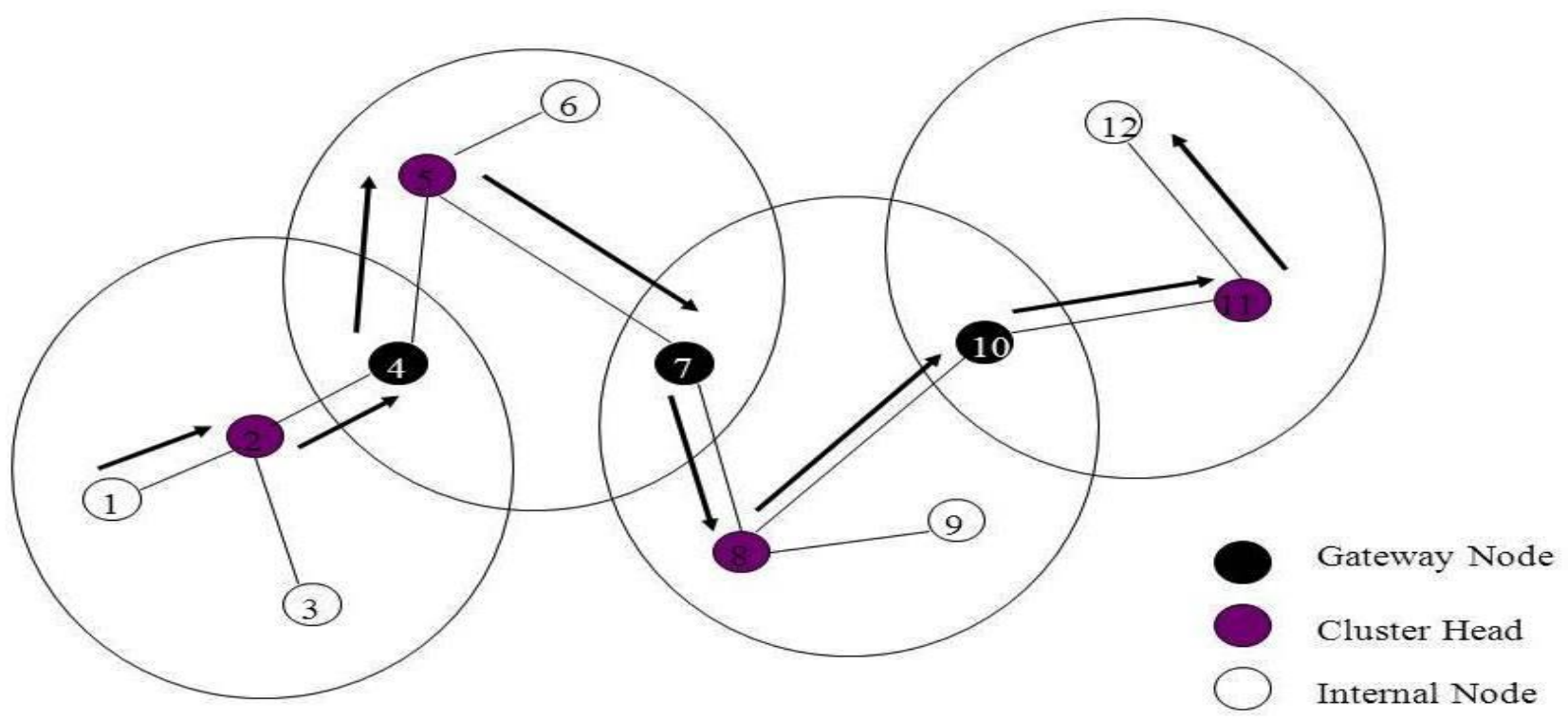

Fig 1: EEHCR Model for routing in between nodes in clusters

\section{PERFORMANCE EVALUATION}

In this section energy efficient hierarchy-based clustering routing (EEHCR) protocol is evaluated and compared it with other available schemes. The simulation is done using ns 2 . To evaluate the performance of EEHCR, the proposed protocol compared with HAR (Hierarchical Anycast Routing Protocol), PEGASIS (Power Efficient Gathering in Sensor Information Systems), LEACH (Low Energy Adaptive Clustering Hierarchy Protocol), TEEN (Threshold sensitive Energy Efficient sensor Network protocol), PEDAP (Power Efficient Data Gathering and Aggregation Routing Protocol) HMRP (Hierarchical Multiple-Choice Routing Path Protocol), PGRP (Pascal Graph Routing Protocol) [12, 15 - 17, 22 - 27]. The following performance metrics are used for comparing different routing protocols:

- Packet Delivery Ratio (PDR): It is the ratio between the number of data packets received and sent.

- Energy consumption: The metric gives the energy consumption of nodes in the event area for transmitting a data packet to sink.

- Average Energy: The metric gives the average of energy of all nodes at the end of simulation.

- Average Latency: The average end-to-end delay observed between transmitting a data packet and receiving it at the destination.

- Network lifetime: This metric gives the time of the first node running out of its energy.

The protocols compared over a set of networks with the number of nodes ranging from 100 to 500 . The aim of the experiment was to measure system lifetime, energy consumption ration, packet delivery ration and latency of each protocol. For the same number of nodes, ten network topologies randomly generate to obtain the average results. All the nodes are set as static nodes. In each network the sensor nodes are distributed in the area of $500 \mathrm{~m}$ X 500m with maximum transmission distance of node set to $50 \mathrm{~m}$ and initial energy of $1 \mathrm{~J}$, the other simulation settings listed in Table 1.

Table 1. The parameters used in the simulation

\begin{tabular}{|c|c|c|c|}
\hline Parameters & Value & Parameters & Value \\
\hline Size of target area & $\begin{array}{c}500 \times 500 \\
\mathrm{~m}^{2}\end{array}$ & $\begin{array}{c}\text { Data packet } \\
\text { size }\end{array}$ & $\begin{array}{c}512 \\
\text { bytes }\end{array}$ \\
\hline $\begin{array}{c}\text { Initial no. of sink } \\
\text { nodes }\end{array}$ & 5 & $\begin{array}{c}\text { Metadata } \\
\text { packet size }\end{array}$ & $\begin{array}{c}25 \\
\text { bytes }\end{array}$ \\
\hline $\begin{array}{c}\text { Initial no. of } \\
\text { sensor nodes }\end{array}$ & 95 & $\begin{array}{c}\text { Maximum } \\
\text { radius, } \mathrm{R}\end{array}$ & $20 \mathrm{~m}$ \\
\hline Initial Energy & $1 \mathrm{~J}$ & $\mathrm{E}_{\text {elec }}$ & $\begin{array}{c}50 \\
\mathrm{~nJ} / \mathrm{bit}\end{array}$ \\
\hline $\begin{array}{c}\text { Transmitting and } \\
\text { Receiving Energy } \\
\left(\mathrm{E}_{\mathrm{Tx}}=\mathrm{E}_{\mathrm{Rx}}\right)\end{array}$ & $50 \mathrm{~nJ} / \mathrm{bit}$ & $\begin{array}{c}\text { Event } \\
\text { duration }\end{array}$ & $50 \mathrm{~min}$ \\
\hline$\Xi_{\mathrm{mp}}$ & $\begin{array}{c}100 \\
\mathrm{pJ} / \mathrm{bit} / \mathrm{m}^{2}\end{array}$ & $\begin{array}{c}\text { Max. no of } \\
\text { rounds }\end{array}$ & 2000 \\
\hline$\xi_{\mathrm{fs}}$ & $10 \mathrm{pJ} / \mathrm{b} / \mathrm{m}^{2}$ & $\begin{array}{c}\text { Max. no of } \\
\text { nodes }\end{array}$ & 500 \\
\hline
\end{tabular}


Performance comparison for Average Energy Dissipation of Routing Protocols

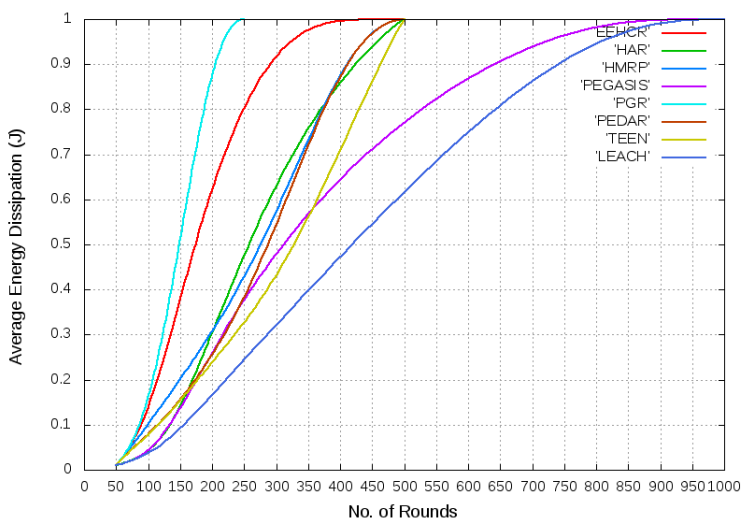

Fig 2(a): Average Energy dissipation of different protocols

Performance comparison for Average Latency of Routing Protocols

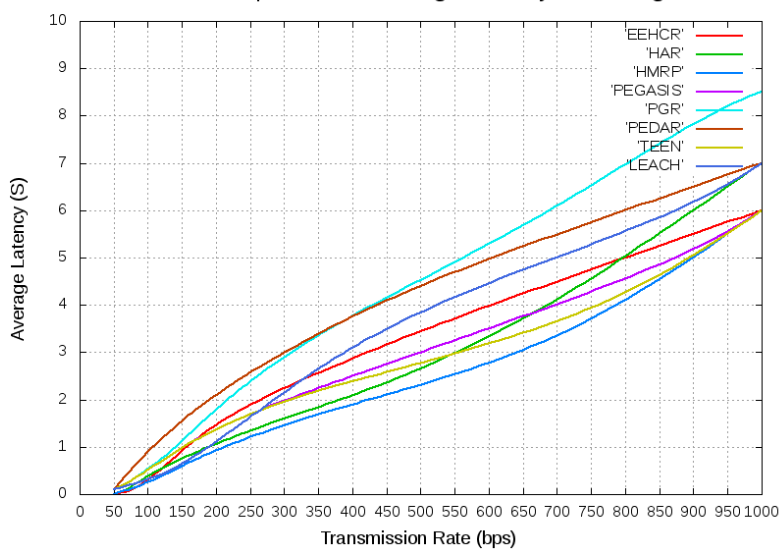

Fig 2(b): Average Latency of different protocols

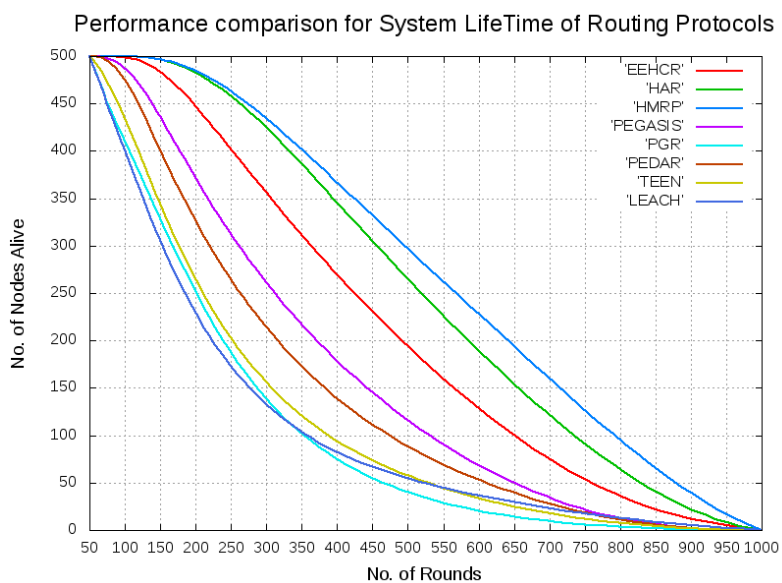

Fig 2(c): The Network Life-Time of different protocols

Fig. 2 shows the comparison of average energy dissipation, average latency and system life-time of different routing protocols. The transmission was increased to evaluate the latency of different protocols. The experiment shows the comparison for 1000 rounds of events for different routing protocols. The result shows that EEHCR is a balanced protocol which can have better throughput and when compared with existing routing protocol overall performance of the routing protocol is better. The result confirms EEHCR delivers the most data packets per unit of energy. EEHCR has better system life-time also when compared with LEACH, PEGASIS, TEEN and HAR. This defines after 500 rounds of packet delivery $50 \%$ of the nodes are still alive which is better than other routing protocols.

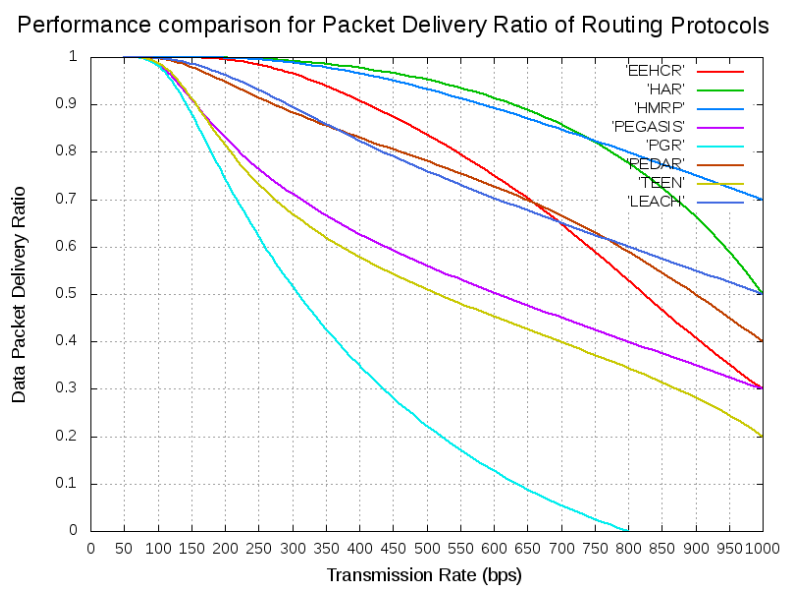

Performance comparison for Packet Delivery Ratio of Routing Protocols

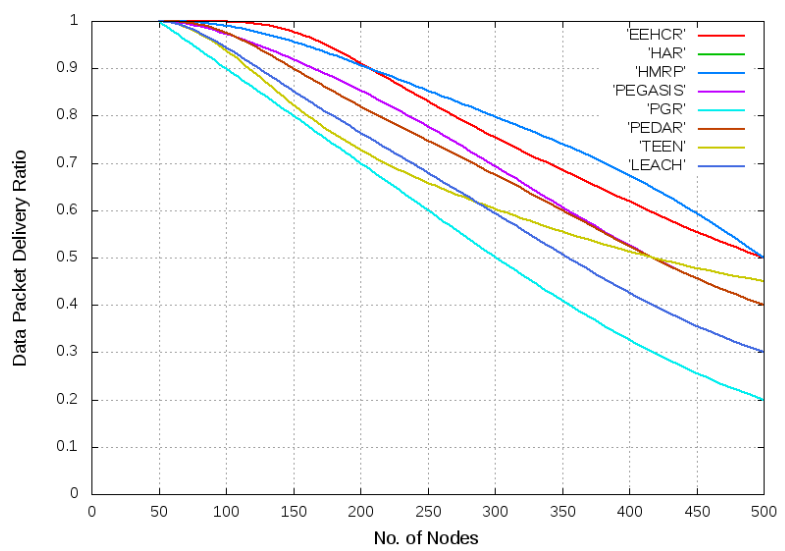

Fig 3: Data Packet Delivery Ratio of different protocols

Fig. 3 shows data packet delivery ratio with increasing number of rounds and transmission rate. The proposed protocol exhibits the rate of packet drops linearly with comparatively low rate.

\section{CONCLUSION}

It is clear from the simulation outcome that by enhancing different properties in the network it is possible to enhance the network life-time and energy efficiency. Energy is one of the major parameter in Wireless Sensor Networks. Routing consumes the largest amount of energy in WSN for routing and in terms of achieving efficient routing mechanism for collecting data packets. Lots of redundant information is available in WSN due to widely deployed nodes but the mechanism is limited to clusters and the hierarchical approaches are different for both inter-cluster and intra-cluster communication due to the pre-defined role of cluster head. This paper has demonstrated the routing strategy of EEHCR protocol and showed how it provides the solution against the dynamic natures of WSN. It also tries to overcome the shortcoming of other protocols in terms of scalability for enhancing network life-time. However, all required performance matrices has not explored. The future work can include exploration of other matrices and mobility of the nodes and incorporate them in the network. 


\section{REFERENCES}

[1] Akyildiz, I.F., Su, W., Sankarasubramaniam, Y. and Cayirci, E., 2002, Wireless sensor networks: a survey, IEEE Computer Networks, vol. 38, no. 4, pp. 393-422.

[2] Akyildiz, I.F., Su, W., Sankarasubramaniam, Y. and Cayirci, E., 2002, A survey on sensor networks, IEEE Communications Magazine, vol. 40, no. 8, Aug 2002, pp. 102-114.

[3] Al-Karaki, J.N.; Kamal, A.E., 2004, Routing techniques in wireless sensor networks: a survey, IEEE Wireless Communications, vol. 11, no. 6, pp. 6-28.

[4] Royer, E. M. and Toh, C. K., 1999, A Review of Current Routing Protocols for ad hoc Mobile Wireless Networks, IEEE Personal Communication, Vol. 6, pp. 46-55.

[5] Hill, J. and Culler, D., 2002, "A Wireless Embedded Sensor Ar-chitecture for System Level Optimization," International Research IRB-TR-02-00N

[6] Doi, S., Ata, S., Kitamura, H., Murata, M. and Miyahara, H., 2003, Protocol design for anycast communication in IPv6 network, IEEE Pacific Rim Conference on Communications, Computers and signal Processing (PACRIM. 2003), vol.1, pp. 470-473.

[7] Anastasi, G., Conti, M., Di Francesco, M., Passarella, A., 2009, "Energy Conservation in Wireless Sensor Networks: A Survey," Elsevier, Ad Hoc Networks, Vol. 7, No. 3, pp. 537-568.

[8] Wen-Wen H., Min Y., Li-Qiong X., Jian W., 2008, "Energy-Efficient Hierarchical Routing Protocol for Wireless Sensor Networks," Pacific-Asia Workshop on Computational Intelligence and Industrial Application (PACIIA '08.), vol.1, pp.640-644.

[9] Barfunga, S.P., Rai, P., Sarma, H.K.D., 2012, "Energy efficient cluster based routing protocol for Wireless Sensor Networks," International Conference on Computer and Communication Engineering (ICCCE), pp.603-607.

[10] Handy, M.J., Haase, M., Timmermann, D., 2002, "Low energy adaptive clustering hierarchy with deterministic cluster-head selection," 4th International Workshop on Mobile and Wireless Communications Network, pp.368372.

[11] Heinzelman, W.R., Chandrakasan, A. and Balakrishnan, H., 2000, Energy-efficient communication protocol for wireless microsensor networks, Proceedings of the 33rd Annual Hawaii International Conference of System Sciences, vol. 2, pp. 10

[12] Lindsey, S. and Raghavendra, C.S., 2002, PEGASIS: Power-efficient gathering in sensor information systems, IEEE Aerospace Conference Proceedings, vol.3, pp. $1125-1130$

[13] Raghunathan, V., Schurgers, C., Sung P. and Srivastava, M.B., 2002, Energy-aware wireless microsensor networks, IEEE Signal Processing Magazine, vol. 19, no. 2, Mar 2002, pp. 40-50

[14] Bandyopadhyay, S., and Coyle, E., 2003, "An EnergyEfficient Hierarchical Clustering Algorithm for Wireless Sensor Net- works," 22nd Annual Joint Conference of the IEEE Computer and Communications (INFOCOM 2003), pp. 1713-1723.
[15] Manjeshwar, A., Agrawal, D.P., 2000, "TEEN: a routing protocol for enhanced efficiency in wireless sensor networks," Proceedings 15th InternationalParallel and Distributed Processing Symposium., pp.2009-2015.

[16] Hüseyin Özgür Tan and Ibrahim Körpeoglu, 2003, Power Efficient Data Gathering and Aggregation in Wireless Sensor Networks, Proc. ACM Conf. on SIGMOD, San Diego, CA, Vol. 32, pp. 66-71.

[17] Thepvilojanapong, N., Tobe, Y. and Sezaki, K., 2005 , HAR: hierarchy-based anycast routing protocol for wireless sensor networks, Proceedings of the 2005 Symposium on Applications and the Internet, pp. 204210.

[18] Yu, Y. and Song, Y., 2010, “An Energy-Efficient ChainBased Routing Protocol in Wireless Sensor Network," International Conference on Computer Application and System Modeling (ICCASM), pp. 486-489.

[19] Jae, D. Y., Kyung T. K.; Bo, Y. J., Hee, Y. Y., 2009, "An Energy Efficient Chain-Based Clustering Routing Protocol for Wireless Sensor Networks," International Conference on Advanced Information Networking and Applications Workshops (WAINA '09.), pp.383-388.

[20] Hong J., Qing-song Y. and Xiao-lei Y., 2011, An improved IPv6 routing lookup algorithm of WSN, Eighth International Conference on Fuzzy Systems and Knowledge Discovery (FSKD), vol.4, pp. 2234-2238.

[21] Sarma, H.K.D., Kar, A., Mall, R., 2011, "Energy efficient routing protocol for Wireless Sensor Networks with Node and Sink mobility," IEEE Sensors Applications Symposium (SAS), pp.239-243.

[22] Jian-Feng Yan, Yuan-Liu Liu, 2011, "Improved LEACH routing protocol for large scale wireless sensor networks routing," International Conference on Electronics, Communications and Control (ICECC), pp.3754-3757.

[23] Yun, L., Nan Y., Weiyi Z., Weiliang Z., Xiaohu Y., Daneshmand, M., 2011, "Enhancing the performance of LEACH protocol in wireless sensor networks," Computer Communications Workshops (INFOCOM WKSHPS), pp.223-228.

[24] Jun, W., Yong-Tao C., Jun-Yuan X., Shi-Fu C., 2011 , "Energy Efficient Backoff Hierarchical Clustering Algorithms for Multi-Hop Wireless Sensor Networks", Journal of Computer Science and Technology.

[25] Ying-Hong, W., Chih-Hsiao T., Hung-Jen M., Kuo-Feng H., 2006, "An energy-efficient hierarchical multiplechoice routing path protocol for wireless sensor networks," IEEE International Conference on Sensor Networks, Ubiquitous, and Trustworthy Computing, vol.1, pp.2.

[26] Guha Neogi, S., 2012, "Routing Method for Wireless Mobile Ad Hoc Networks using Pascal Graph Topology," International Journal of Research in Computer Engg. and Electronics, vol.1, no.3.

[27] Panwar, D. and Guha Neogi, S., 2013, "Design of Energy Efficient Routing Algorithm for Wireless Sensor Network (WSN) Using Pascal Graph," CSCP: Journal of Computer Science \& Information Technology (CS \& IT), vol.3, no. 2 , pp. $175-189$. 